\title{
Long-circulating and target-specific distributions of cyanine 5.5-labeled hyaluronic acid nanoparticles in mouse organs during 28 days after a single administration
}

\author{
Tae Sik Yun ${ }^{1, \dagger}$, Chunmei Lin ${ }^{2, \dagger}$, Jung-Min Yon ${ }^{1}$, Seul Gi Park ${ }^{1}$, Lee Wha Gwon ${ }^{1}$, Jong-Geol Lee ${ }^{1}$, In-Jeoung Baek ${ }^{3}$, \\ Sang-Seop Nahm ${ }^{4}$, Beom Jun Lee ${ }^{1}$, Young Won Yun ${ }^{1}$, Sang-Yoon Nam ${ }^{1, *}$ \\ ${ }^{1}$ College of Veterinary Medicine and Veterinary Medical Center, Chungbuk National University, Cheongju 28644, Korea \\ ${ }^{2}$ College of Chinese Medicinal Materials, Jilin Agricultural University, Changchun 130-118, China \\ ${ }^{3}$ Asan Institute for Life Sciences, University of Ulsan College of Medicine, Seoul 05505, Korea \\ ${ }^{4}$ College of Veterinary Medicine, Konkuk University, Seoul 05029, Korea
}

(Received: October 6, 2018; Revised: December 6, 2018; Accepted: December 10, 2018)

\begin{abstract}
Although hyaluronic acid (HA) has been developed as a nanoparticle (NP; 320-400 nm) for a drug delivery system, the tissue targeting efficacy and the pharmacokinetics of HA-NPs are not yet fully understood. After a dose of $5 \mathrm{mg} / \mathrm{kg}$ of cyanine 5.5-labeled HA-NPs or HA-polymers was intravenously administrated into mice, the fluorescence was measured from $0.5 \mathrm{~h}$ to 28 days. The HA-NPs fluorescence was generally stronger than that of HA-polymers, which was maintained at a high level over 7 days in vivo, after which it gradually decreased. Upon ex vivo imaging, liver, spleen, kidney, lung, testis and sublingual gland fluorescences were much higher than that of other organs. The fluorescence of HA-NPs in the liver, spleen and kidney was highest at $30 \mathrm{~min}$, where it was generally maintained until $4 \mathrm{~h}$, while it drastically decreased at 1 day. However, the fluorescence in the liver and spleen increased sharply at 7 days relative to 3 days, then decreased drastically at 14 days. Conversely, the fluorescence of HA-polymers in the lymph node was higher than that of HA-NPs. The results presented herein may have important clinical implications regarding the safety of as self-assembled HA-NPs, which can be widely used in biomedical applications.
\end{abstract}

Keywords: drug delivery systems, hyaluronic acid, nanoparticles, tissue distribution, toxicokinetics

\section{Introduction}

A variety of nanoparticles (NPs) have been produced with the development of nanotechnology in recent years [13]. Nanotechnology has already been applied clinically for pharmaceutical drug delivery systems (DDSs) and development of therapies for various diseases and disorders [1]. In particular, various self-assembled NPs have been produced to deliver diagnostic and/or therapeutic drugs to target sites, promoting early detection and efficient treatment of cancer [5]. Furthermore, NPs have been widely investigated in many areas, such as the predictive paradigm for oxidative stress, DNA array detection, bio-bar codes for detection of proteins, gene delivery to cells, scaffolds for tissue engineering and lymphatic targeting materials. Therefore, the number of newly generated nanomaterials has been continuously increasing along with their uses [6, 11, 17, 18, 21, 24, 27].

Despite this enormous increase in the prevalence of engi- neered nanomaterials, little is known about their toxicological effects on humans and the environment [14]. Moreover, even though much attention and effort is being put into development of nano-based therapeutic uses, the level of translational clinical use remains restrictive because of uncertainty in the toxicological effects of the NPs [11]. Specifically, the behavior of nano-sized particles in natural states and their consequent ecological impacts are still poorly understood [20]. Many studies have been conducted to develop new DDSs using biopolymers and synthetic polymers with a variety of functions and performance to reduce the side effects of existing biomedical therapies, optimize the efficiency and effects of the medication and efficiently deliver the minimum concentration of the drug required for treating diseases [13]. One material that satisfies those needs is hyaluronic acid (HA), which has diverse biomedical uses [25]. These self-assembled NPs, composed of biocompatible and amphiphilic polymer conjugates, have shown prolonged

\footnotetext{
*Corresponding author

Tel: +82-43-261-2596, Fax: +82-43-271-3246

E-mail: synam@cbu.ac.kr

The first two authors contributed equally to this work.
} 
circulation in the blood stream after systemic administration in vivo [15].

Hyaluronic acid, which is also referred to as hyaluronan, is a naturally existing polysaccharide belonging to the family of glycosaminoglycan. This acid consists of repetitive D-glucuronic acid- $\beta-(1,3)-N$-acetyl-D-glucosamine disaccharide units connected together through $\beta(1,4)$-glycosidic bonds [2]. HA is an extracellular and cellular surface-related polysaccharide that is generally referred to as biological 'goo' that is involved in lubricating joints or holding together gel-like connective tissues [12]. As a result, it is generally found in various organs and tissues such as the extracellular matrix and synovial fluids of the body. Moreover, it has been studied as a targeting moiety of drug delivery agents or NPs for tumor treatment since it can specifically locate and stick to a variety of tumor cells that express high levels of CD44, an HA receptor [5]. Hyaluronic acid has been found in a broad range of molecular weights, generally from 20 to $4,000 \mathrm{kDa}$, and this wide range of weights enables its application in therapeutic formulations [25]. HA is a substance of increasing importance to biomaterials disciplines that is finding applications in various fields, such as DDS, cosmetic materials and tissue culture scaffolds [6].

It is well known that the biodistribution and accumulation of NPs are affected by their physicochemical characteristics, such as chemical properties of the surface, molecular weight, size, shape, charge and density. However, its detailed in vivo properties, such as tissue targeting efficacy and the pharmacokinetics of HA-modified macromolecular prodrugs or NPs are not yet fully understood. Therefore, in this study, we evaluated the distribution and toxicity of HA-NPs and HApolymers in tissues after intravenous application and investigated the time-dependent fates of HA-NPs and HA-polymers in the animal body over 28 days. Overall, the results of this study provide pharmacokinetics and safety information regarding the use of HA-NPs and HA-polymers as a drug delivery agent.

\section{Materials and Methods}

\section{Preparation of HA}

Completely prepared HA-NPs and polymers were obtained from Dr. Kwangmeyung Kim, Korea Institute of Science and Technology (Seoul, Korea). The preparation and conjugation of HA used in the experiments was conducted as previously described [3]. Briefly, sodium hyaluronate $\left(\mathrm{M}_{\mathrm{w}}=2.344 \times 10^{5}\right)$ was purchased from Lifecore Biomedical (USA). Sodium hyaluronate was dialyzed against distilled water, which was followed by lyophilization, after which it was used. Cyanine 5.5 (Cy5.5) was purchased from Amersham Biosciences (USA). HA-5 $\beta$-cholanic acid conjugates (HA-CA conjugates) with an amphiphilic property were generated by chemical process or by conjugation of hydrophobic bile acid (5 $\beta$ cholanic acid) to the hydrophilic HA backbone via formation of amide. To generate the HA-NPs, phosphate-buffered saline (PBS; pH 7.4) was used to dissolve HA-CA conjugates. This solution was sonicated three times for 2 min each using a probe-type sonifier (VCX-750; Sonics \& Materials, USA) at $90 \mathrm{~W}$. The particle diameter of the HA-NPs was measured by applying dynamic light scattering with a helium ion laser system (Laser Model 127-35; Spectra-Physics, USA). For animal tests, HA-NPs and HA-polymers were labeled with Cy5.5 to show their biodistribution and accumulation in vivo. In the presence of 1-hydroxybenzotriazole and 1-ethyl-3-(3-dimethylaminopropyl)carbodiimide, HA-CA conjugates were chemically treated with adipic acid dihydrazidemodified Cy5.5. The concentration of Cy5.5 molecules attached to conjugate was fixed to $3.2 \mathrm{wt} \%$, as measured using a FLx800 fluorescence reader (BioTek, USA) at 680 $\mathrm{nm}$. The average size of the HA-NPs was $320-400 \mathrm{~nm}$, which is similar to that of those that have been applied for clinical applications.

\section{Animals}

Six-week-old ICR and hairless (Hos: HR-1) male mice were purchased from Korea animal technology (Korea) and Central Laboratory Animal Inc. (Korea), respectively. The mice were housed under the following conditions: an ambient temperature of $20^{\circ} \mathrm{C} \pm 2{ }^{\circ} \mathrm{C}$, relative humidity of $50 \pm$ $10 \%$, air ventilation rate of 10 cycles per hour, and a $12: 12 \mathrm{~h}$ light-dark cycle. The animals were fed standard mouse chow (Samyang, Korea) and tap water ad libitum throughout the experimental period. Mice weighing $20 \pm 2 \mathrm{~g}$ were used for this study. After acclimatization for one week, six mice were assigned to each group (time-dependent group). All experiments were approved by the Chungbuk National University Animal Care Committee and conducted according to the Guide for Care and Use of Animals (Chungbuk National University Animal Care Committee, CBNUA-608-13-01).

\section{Ex vivo biodistribution of free $\mathrm{Cy} 5.5$}

For free Cy5.5 imaging, a dose of $0.1 \mathrm{mg} / \mathrm{kg}$ of free Cy5.5 was injected into the tail vein of 7-week-old hairless male mice. A total of 6 mice were used in this control experiment. After injection of free Cy5.5, the mice were anesthetized and target organs were separated at $0.5 \mathrm{~h}, 2 \mathrm{~h}, 4 \mathrm{~h}, 1$ day (d), $3 \mathrm{~d}$, $7 \mathrm{~d}, 14 \mathrm{~d}$, and $28 \mathrm{~d}$. Target organs were the liver, kidney, heart, lung, stomach, lymph node, testis, small intestine and large intestine. Fluorescence images of dissected organs were obtained as a function of time using an IVIS 200 in vivo imaging system (Xenogen, USA). For accurate analysis of results, the biodistribution of free Cy5.5 was quantified by measuring the fluorescence intensity (photons $/ \mathrm{sec} / \mathrm{cm}^{2}$ ) from an ex vivo image.

\section{In vivo biodistribution of Cy5.5-labeled HA-NPs and HA-polymers}

There were two experimental groups, a Cy5.5-labeled HANPs treated-group and a Cy5.5-labeled HA-polymers treatedgroup. Each experimental group contained a total 56 hairless 
male mice, and seven mice were assigned to each timedependent subgroup $(0.5 \mathrm{~h}, 2 \mathrm{~h}, 4 \mathrm{~h}, 1 \mathrm{~d}, 3 \mathrm{~d}, 7 \mathrm{~d}, 14 \mathrm{~d}$, and $28 \mathrm{~d}$ ). One of seven mice in the subgroups was used as a control mouse to compare with the results of HA treatedmice. The control mouse was administrated saline alone instead of Cy5.5-labeled HA-NPs or HA-polymers. Therefore, six mice per subgroup were used to analyze the results. Cy5.5-labeled HA-NPs or HA-polymers were injected into the tail vein of 7-week-old hairless male mice at a dose of $5 \mathrm{mg} / \mathrm{kg}$. After injection of Cy5.5-labeled HA-NPs or HApolymers, the mice were anesthetized and fluorescence images were captured using an IVIS 200 in vivo imaging system (Xenogen). To estimate the time-dependent fates of HA-NPs or HA-polymers, the total change in fluorescence in the whole body was measured at $0.5 \mathrm{~h}, 2 \mathrm{~h}, 4 \mathrm{~h}, 1 \mathrm{~d}, 3 \mathrm{~d}, 7 \mathrm{~d}$, $14 \mathrm{~d}$, and $28 \mathrm{~d}$. Finally, the amount and alteration of the fluorescence image in response to HA-NPs were compared to those observed in the HA-polymers and non-treated (salineinjected) groups to identify the effects of particle size.

\section{Ex vivo distribution of HA-NPs and HA-polymers}

Mice were prepared and assigned to each group as for investigation of the in vivo distribution of Cy5.5-labeled HA-
NPs or HA-polymers. Similarly, control mice were administrated saline alone instead of Cy5.5-labeled HAs. Cy5.5labeled HA-NPs or HA-polymers were injected into the tail vein of 7-week-old ICR male mice at a dose of $5 \mathrm{mg} / \mathrm{kg}$. After injection of Cy5.5-labeled HA-NPs or HA-polymers, the mice were anesthetized and target organs were separated at $0.5 \mathrm{~h}, 2 \mathrm{~h}, 4 \mathrm{~h}, 1 \mathrm{~d}, 3 \mathrm{~d}, 7 \mathrm{~d}, 14 \mathrm{~d}$, and $28 \mathrm{~d}$. The target organs were the brain, sublingual gland, thymus, heart, lung, liver, spleen, kidney, testis, epididymis and adrenal gland. Fluorescence images of dissected organs were obtained with an IVIS 200 in vivo imaging system (Xenogen).

\section{Fluorescence intensity measurement from ex vivo image} For accurate analysis of the results, the biodistributions of HA were quantified by measuring the fluorescence intensity (photons $/ \mathrm{sec} / \mathrm{cm}^{2}$ ) from an ex vivo image. The target organs were the brain, lymph node, thymus, heart, lung, liver, spleen, kidney, testis, epididymis and adrenal gland. All values are expressed as a function of time.

\section{Statistical analysis}

Statistical differences between groups were analyzed by one-way analysis of variance (ANOVA) followed by Tukey's

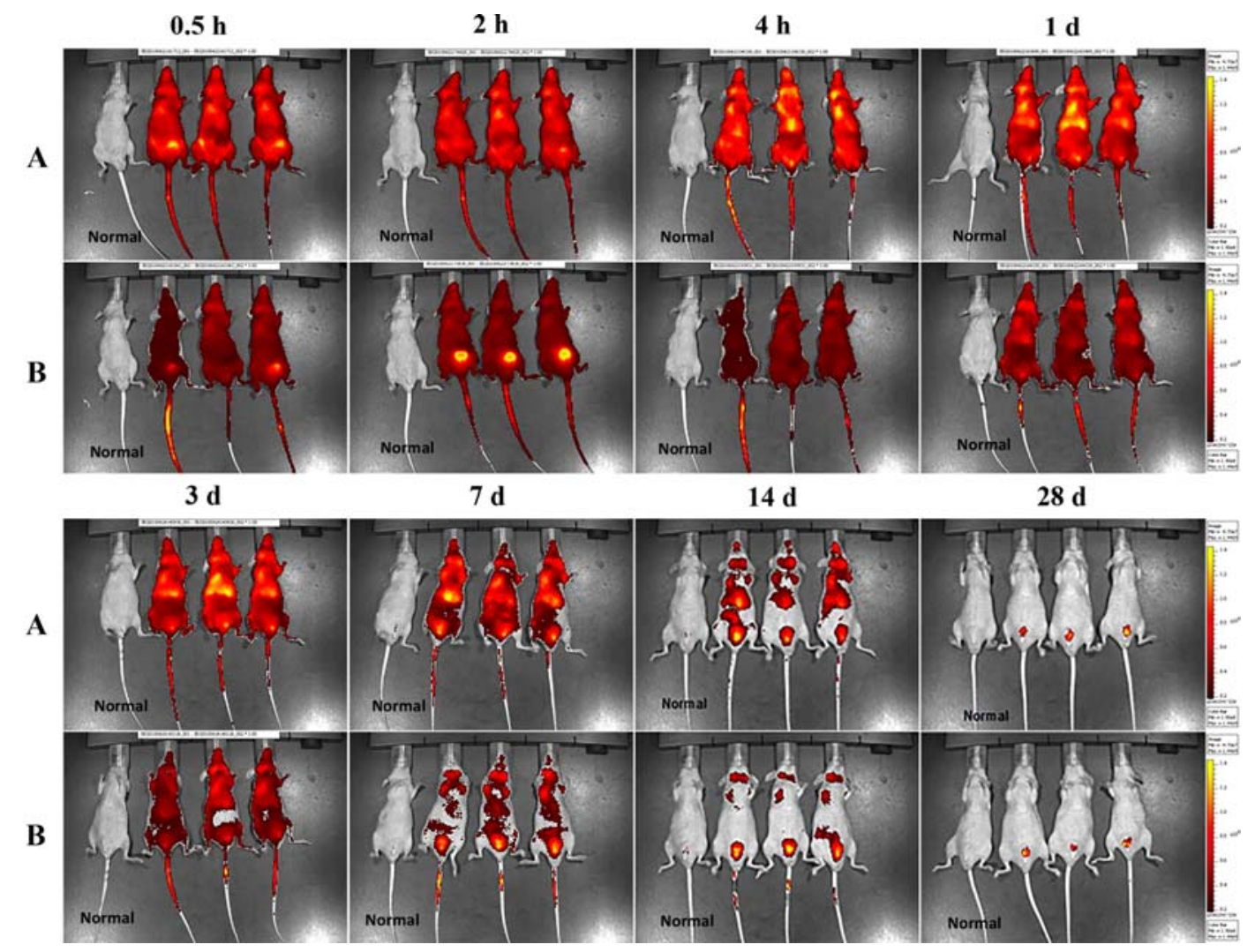

Fig. 1. In vivo fluorescence images of Cy5.5-labeled HA-NPs (A) and Cy5.5-labeled HA-polymers (B) in the whole body ( $\mathrm{n}=6$ ) at $0.5 \mathrm{~h}, 2 \mathrm{~h}, 4 \mathrm{~h}, 1$ day (d), $3 \mathrm{~d}, 7 \mathrm{~d}, 14 \mathrm{~d}$, and $28 \mathrm{~d}$. Tail vein injection of Cy5.5-labeled HA-NPs or HA-polymers $(5 \mathrm{mg} / \mathrm{kg})$ in normal hairless mouse. The fluorescence of HA-NPs was much stronger than that of HA-polymers and was maintained longer within the experimental period relative to HA-polymers. The liver and urinary bladder were the main organs that showed strong fluorescence in both groups. Becoming yellow indicates a higher intensity of fluorescence at $680 \mathrm{~nm}$. Normal, untreated mice. 
multiple comparison test. In addition, statistical differences among times in the same group were analyzed by independent $t$-tests. Statistical significance was established at $p<$ 0.05 . The ex vivo biodistribution of free Cy5.5 was expressed as the means \pm SD for groups of six animals. Data describing the fluorescence intensity of HAs from ex vivo images were expressed as the means \pm SE for groups of six animals. All analyses were conducted using SPSS for Windows (ver. 23.0; IBM, USA).

\section{Results}

Ex vivo imaging of free Cy5.5

The liver, lung, stomach and kidney were the main organs in which free Cy5.5 was distributed and accumulated. In addition, free Cy5.5 was detected at higher levels in these organs on the initial day than at other times. Among the total organs, free Cy5.5 was primarily accumulated in the liver. The fluorescence of the liver showed the highest level at $0.5 \mathrm{~h}\left(65.2 \times 10^{6} \pm 0.0120 \times 10^{6} / \mathrm{photons} / \mathrm{sec} / \mathrm{cm}^{2}\right)$, and was reduced by half at $1 \mathrm{~h}\left(32.4 \times 10^{6} \pm 0.0056 \times 10^{6} /\right.$ photons/ $\left.\mathrm{sec} / \mathrm{cm}^{2}\right)$, then continued to decrease greatly until $1 \mathrm{~d}(5.5 \times$ $\left.10^{6} \pm 0.0010 \times 10^{6} / \mathrm{photons} / \mathrm{sec} / \mathrm{cm}^{2}\right)$. One day after the administration of free Cy5.5, it was excreted from the liver very slowly. However, the fluorescence of the liver was maintained at levels much higher than that of other organs during the first day. In kidney, lung and stomach, the levels of fluorescence were much lower than those of the liver, but were

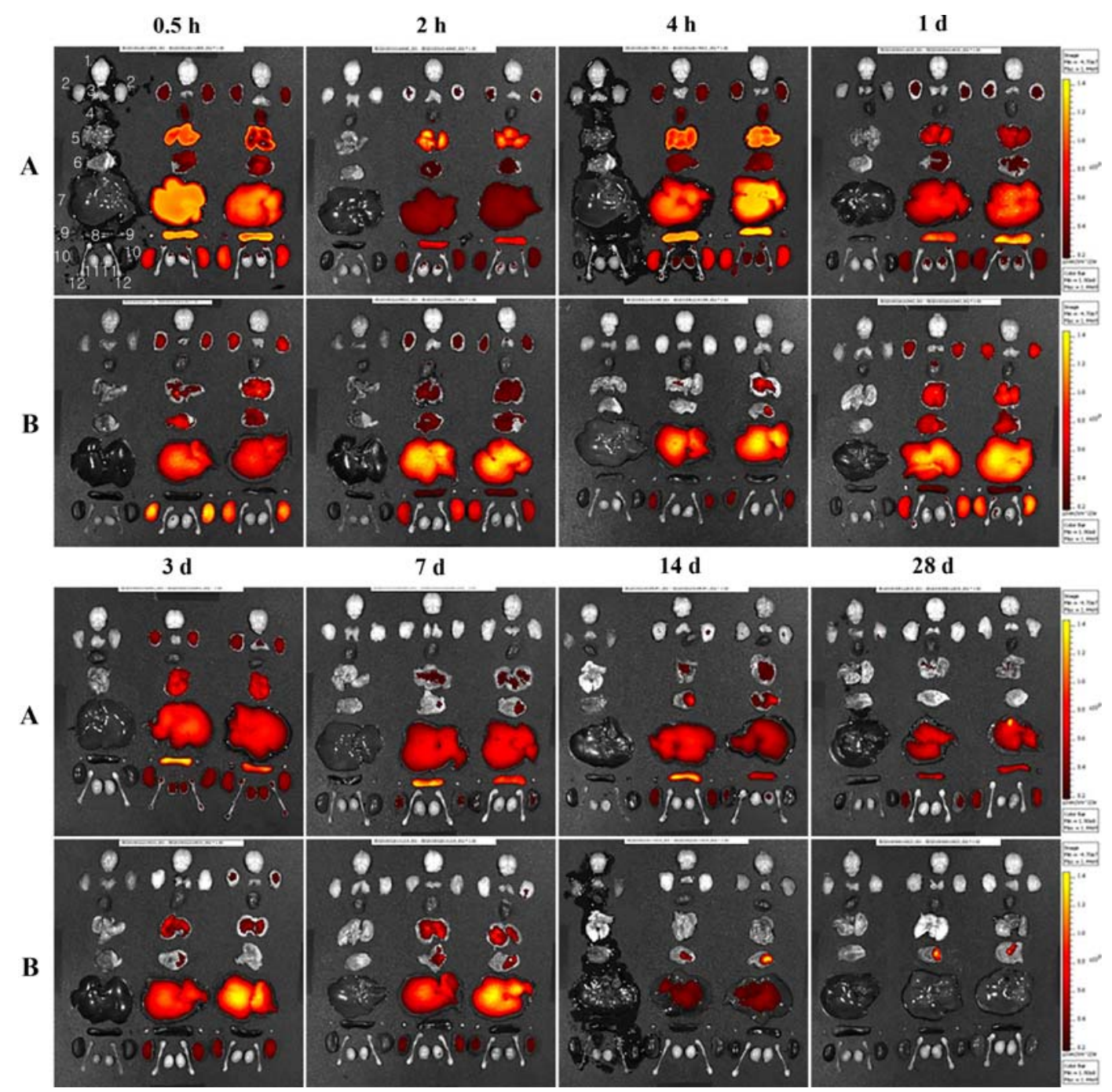

Fig. 2. Ex vivo fluorescence images of Cy5.5-labeled HA-NPs (A) and Cy5.5-labeled HA-polymers (B) in different organs (brain, thymus, sublingual gland, heart, lung, liver, spleen, kidney, adrenal gland, testis and epididymis; $\mathrm{n}=6$ ) at $0.5 \mathrm{~h}, 2 \mathrm{~h}, 4 \mathrm{~h}, 1 \mathrm{~d}, 3 \mathrm{~d}, 7 \mathrm{~d}$, $14 \mathrm{~d}$, and $28 \mathrm{~d}$. Tail vein injection of Cy5.5-labeled HA-NPs or HA-polymers $(5 \mathrm{mg} / \mathrm{kg})$ in normal ICR mice. Generally, the overall fluorescence of HA-NPs was stronger than that of HA-polymers and was maintained longer during the experimental period. The liver, spleen, kidney and lung were the main organs that showed strong fluorescence in both groups. Becoming yellow indicates a higher intensity of fluorescence at $680 \mathrm{~nm}$. 1, brain; 2, sublingual glands; 3, thymus; 4, heart; 5, lung; 6, stomach; 7, liver; 8, spleen; 9, adrenal glands; 10, kidneys; 11, testes; 12, epididymes. 
higher than those of other organs during the first $4 \mathrm{~h}$. The fluorescence of the other organs remained low throughout the experimental period and decreased slowly with time (data not shown).

In vivo imaging of Cy5.5-labeled HA-NPs and HApolymers throughout the body

As shown in Figure 1, evaluation of the whole image of the body revealed that HA-treated groups showed significant differences compared to the normal group, but the distribution of Cy5.5-labeled HAs among organs was not clearly confirmed. Generally, the fluorescence of the HA-NPs groups (Fig. 1A) was much stronger than that of the HA-polymers (Fig. 1B) within the experimental period. The presence of Cy5.5-labeled HA-NPs was confirmed to be at a high level throughout the body, especially in the urinary bladder at $0.5 \mathrm{~h}$ after intravenous injection. The fluorescence in the liver was detected at a high level at $4 \mathrm{~h}$. The high level of this fluorescence throughout the body was maintained until $7 \mathrm{~d}$, and it started to slowly decrease at $14 \mathrm{~d}$. However, it remained in the liver, lung and urinary bladder continuously after that time, and the last organ that showed fluorescence was the urinary bladder. Similarly, the presence of Cy5.5-labeled HApolymers was confirmed to be at a high level throughout the body at $0.5 \mathrm{~h}$ after intravenous injection, but it showed a much lower level of fluorescence than the HA-NPs group. The urinary bladder was the brightest organ during the initial $30 \mathrm{~min}$. Specifically, fluorescence was detected at the highest level in the urinary bladder at $2 \mathrm{~h}$ and in the liver at $1 \mathrm{~d}$. The high level of this fluorescence throughout the body was maintained until $1 \mathrm{~d}$ and it decreased slowly at $3 \mathrm{~d}$. However, it remained in the lung and urinary bladder after that time and, with fluorescence remaining in the urinary bladder longest.

\section{Ex vivo imaging of Cy5.5-labeled HA-NPs and HA- polymers in tissues}

Generally, the overall fluorescence of HA-NPs (Fig. 2A) in organs was stronger and maintained for longer than HApolymers (Fig. 2B). The presence of Cy5.5-labeled HA-NPs was confirmed to be at a high level in the lung, liver, spleen and kidney at $0.5 \mathrm{~h}$ after intravenous injection. The fluorescence in those organs was maintained until $4 \mathrm{~h}$, and it started
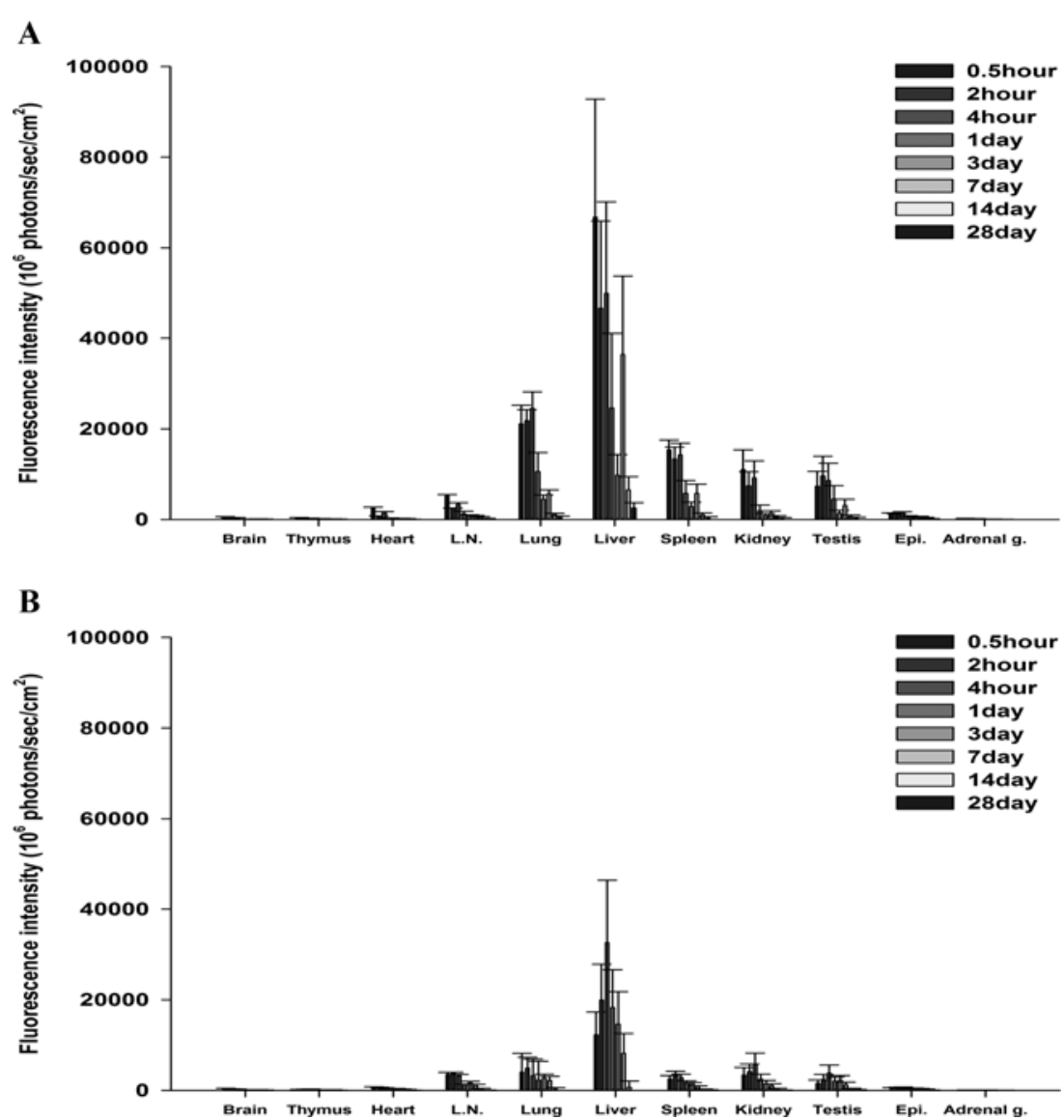

Fig. 3. Fluorescence intensity $\left(10^{6}\right.$ photons $\left./ \mathrm{sec} / \mathrm{cm}^{2}\right)$ of total organs from $0.5 \mathrm{~h}$ to $28 \mathrm{~d}$ after injection of $0.5 \mathrm{mg} / \mathrm{kg}$ of Cy5.5-labeled HA-NPs (A) or Cy5.5-labeled HA-polymers (B). The main organs that showed a high intensity of fluorescence were the liver, lung, spleen, kidney, testis and sublingual gland in both groups. Generally, the intensity of fluorescence peaked within $4 \mathrm{~h}$, then decreased gradually with time. The intensity of Cy5.5-labeled HA-NPs (A) was much stronger than that of Cy5.5-labeled HA-polymers (B). Data are expressed as the means $\pm \mathrm{SE}(\mathrm{n}=6)$. 
to decrease at $1 \mathrm{~d}$; however, it remained in the liver, spleen and kidney throughout the experiment. Similarly, the presence of Cy5.5-labeled HA-polymers was confirmed to be at a high level in the sublingual gland, testis, lung, liver and kidney at $0.5 \mathrm{~h}$ after intravenous injection. With the exception of the liver, the main organs started to show decreased fluorescence at $2 \mathrm{~h}$. However, the fluorescence of liver increased at $2 \mathrm{~h}$ and was maintained until $1 \mathrm{~d}$. The fluorescence of the lung, liver and kidney was detected at $7 \mathrm{~d}$, but the last organs in which fluorescence remained were the liver and lung. While the fluorescence of HA-NPs was maintained in some organs until $28 \mathrm{~d}$, nearly no fluorescence of HA-polymers was detected in any organs at $28 \mathrm{~d}$. The brain was the only organ in which fluorescence was not detected in either group.

\section{Fluorescence intensity from ex vivo imaging in main} organs

Figure 3 shows the overall alteration of fluorescence inten-
A

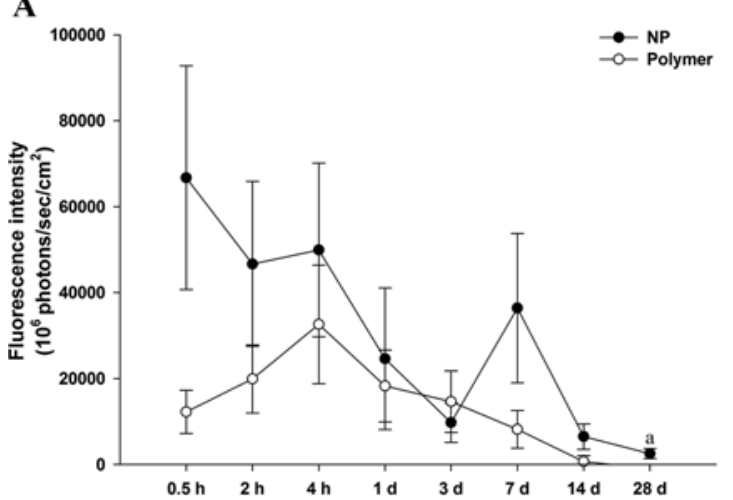

C

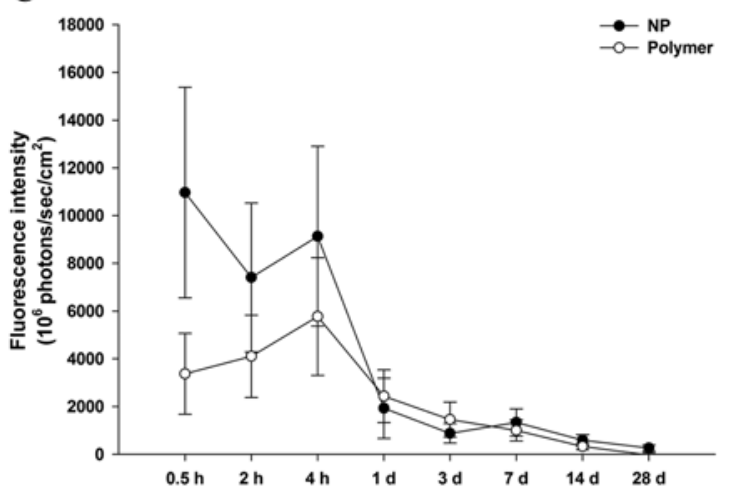

$\mathbf{E}$

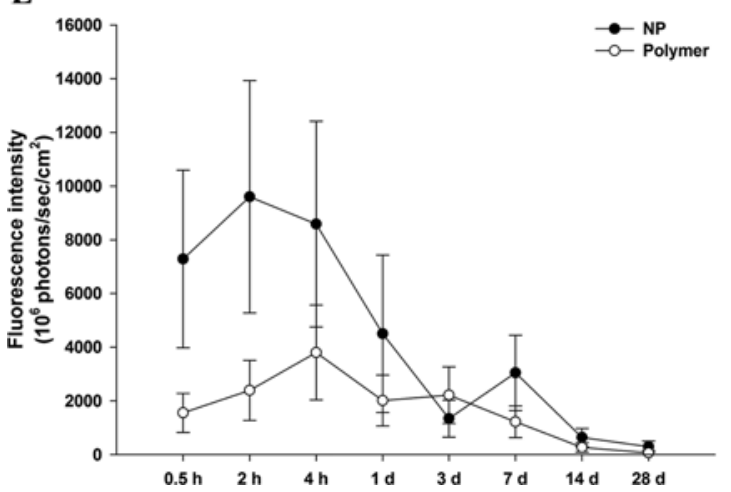

B

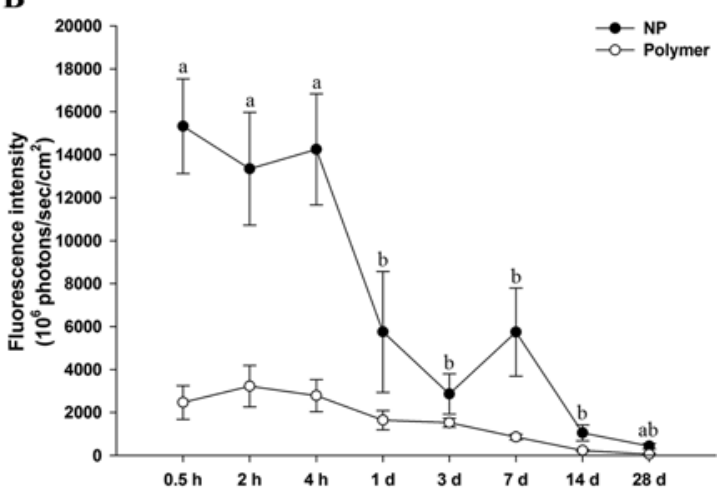

D

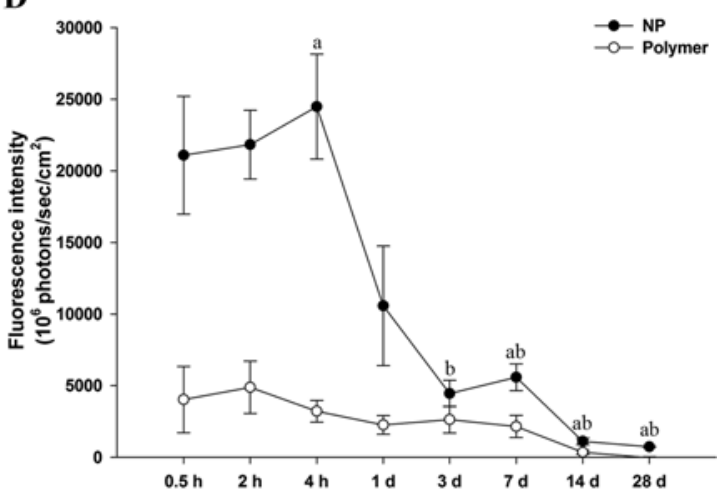

F

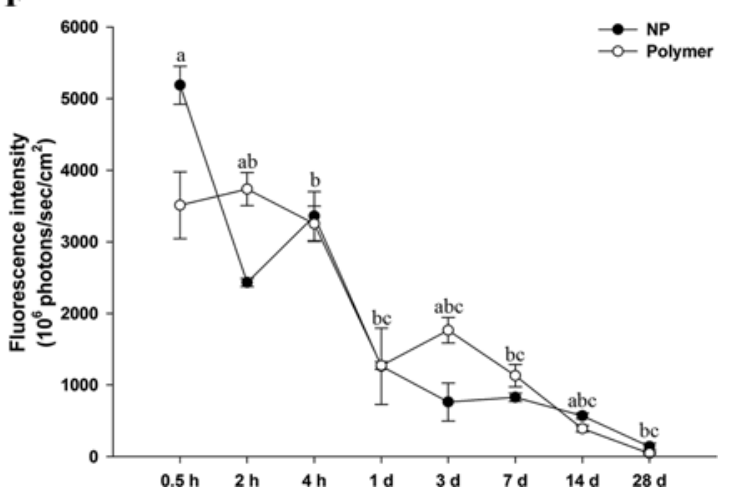

Fig. 4. Fluorescence intensity $\left(10^{6}\right.$ photons $\left./ \mathrm{sec} / \mathrm{cm}^{2}\right)$ in the liver (A), spleen (B), kidney (C), lung (D), testis (E) and lymph node (F) from $0.5 \mathrm{~h}$ to $28 \mathrm{~d}$ after injection of $0.5 \mathrm{mg} / \mathrm{kg}$ of Cy5.5-labeled HA-NPs or Cy5.5-labeled HA-polymers. The intensities of Cy5.5labeled HA-NPs were much stronger level than those of Cy5.5-labeled HA-polymers. Generally, the intensity of fluorescence peaked within $4 \mathrm{~h}$, then decreased gradually thereafter. However, the lymph node showed more powerful intensities of fluorescence in HApolymers within the experimental period than HA-NPs. ${ }^{\mathrm{a}} p<0.05$ between HA-NPs and HA-polymers. ${ }^{\mathrm{b}, \mathrm{c}} p<0.05$ compared with the intensity of $0.5 \mathrm{~h}$. Data are expressed as the means $\pm \operatorname{SE}(n=6)$. 
sity for total organs in HA-NPs (Fig. 3A) and HA-polymers (Fig. 3B) during the experimental periods. Generally, the level of fluorescence peaked from 0.5 to $4 \mathrm{~h}$ in both groups, after which it started to decrease. The primary organs that showed more fluorescence intensity were the liver, spleen, kidney, lung, testis and lymph node.

In the liver (Fig. 3A), the fluorescence intensity of Cy5.5labeled HA-NPs showed the highest level at $0.5 \mathrm{~h}(66,740 \times$ $10^{6} \pm 26,065 \times 10^{6} /$ photons $/ \mathrm{sec} / \mathrm{cm}^{2}$ ), which was generally maintained at a high level until $4 \mathrm{~h}$. This intensity of fluorescence decreased significantly at $1 \mathrm{~d}\left(24,608 \times 10^{6} \pm 16,471 \times\right.$ $10^{6} / \mathrm{photons} / \mathrm{sec} / \mathrm{cm}^{2}$ ) and decreased gradually from 1 to $28 \mathrm{~d}$, except around $7 \mathrm{~d}$. The intensity of fluorescence at $7 \mathrm{~d}(36,385 \times$ $\left.10^{6} \pm 17,379 \times 10^{6} / \mathrm{photons} / \mathrm{sec} / \mathrm{cm}^{2}\right)$ sharply increased by more than that of $3 \mathrm{~d}\left(9,734 \times 10^{6} \pm 4,574 \times 10^{6} / \mathrm{photons} / \mathrm{sec} / \mathrm{cm}^{2}\right)$, then decreased drastically at $14 \mathrm{~d}\left(6,499 \times 10^{6} \pm 2,954 \times 10^{6} /\right.$ photons $/ \mathrm{sec} / \mathrm{cm}^{2}$ ). The fluorescence intensity of Cy5.5-labeled HA-polymers in the liver increased gradually after administration and showed the highest level at $4 \mathrm{~h}\left(32,605 \times 10^{6} \pm\right.$ $13,799 \times 10^{6} /$ photons $\left./ \mathrm{sec} / \mathrm{cm}^{2}\right)$, which decreased gradually until $28 \mathrm{~d}$.

As shown in Figure 4B, the fluorescence intensity of Cy5.5-labeled HA-NPs in the spleen was highest level at $0.5 \mathrm{~h}\left(15,327 \times 10^{6} \pm 2208 \times 10^{6} / \mathrm{photons} / \mathrm{sec} / \mathrm{cm}^{2}\right)$, and was generally maintained at a high level until $4 \mathrm{~h}$. This intensity of fluorescence decreased significantly at $1 \mathrm{~d}\left(5,754 \times 10^{6} \pm\right.$ $\left.2,815 \times 10^{6} / \mathrm{photons} / \mathrm{sec} / \mathrm{cm}^{2}\right)$, and decreased gradually from 1 to $28 \mathrm{~d}$, except around $7 \mathrm{~d}$. The fluorescence intensity at $7 \mathrm{~d}$ $\left(5,743 \times 10^{6} \pm 2,051 \times 10^{6} / \mathrm{photons} / \mathrm{sec} / \mathrm{cm}^{2}\right)$ sharply increased more than that at $3 \mathrm{~d}\left(2,864 \times 10^{6} \pm 934 \times 10^{6} / \mathrm{photons} / \mathrm{sec} /\right.$ $\left.\mathrm{cm}^{2}\right)$, then decreased drastically at $14 \mathrm{~d}\left(1,052 \times 10^{6} \pm 370 \times\right.$ $10^{6} / \mathrm{photons} / \mathrm{sec} / \mathrm{cm}^{2}$ ). The fluorescence intensity of Cy5.5labeled HA-polymers in the spleen increased gradually after administration, and showed the highest level at $2 \mathrm{~h}(3,224 \times$ $\left.10^{6} \pm 959 \times 10^{6} / \mathrm{photons} / \mathrm{sec} / \mathrm{cm}^{2}\right)$, then decreased gradually until $28 \mathrm{~d}$.

In the kidney (Fig. 4C), the fluorescence intensity of Cy5.5-labeled HA-NPs showed the highest level at $0.5 \mathrm{~h}$ $\left(10,965 \times 10^{6} \pm 4,414 \times 10^{6} / \mathrm{photons} / \mathrm{sec} / \mathrm{cm}^{2}\right)$, and was generally maintained at a high level until $4 \mathrm{~h}$. This intensity of fluorescence decreased significantly at $1 \mathrm{~d}\left(1,926 \times 10^{6} \pm 1,263\right.$ $\left.\times 10^{6} / \mathrm{photons} / \mathrm{sec} / \mathrm{cm}^{2}\right)$, then decreased gradually from 1 to $28 \mathrm{~d}$. The fluorescence intensity of Cy5.5-labeled HA-polymers in the kidney increased gradually after administration and showed the highest level at $4 \mathrm{~h}\left(5,771 \times 10^{6} \pm 2,467 \times\right.$ $\left.10^{6} / \mathrm{photons} / \mathrm{sec} / \mathrm{cm}^{2}\right)$, then decreased gradually until $28 \mathrm{~d}$.

In the lung (Fig. 4D), the fluorescence intensity of Cy5.5labeled HA-NPs increased significantly after administration, showing the highest level at $4 \mathrm{~h}\left(24,486 \times 10^{6} \pm 3,667 \times 10^{6}\right.$ \% photons $/ \mathrm{sec} / \mathrm{cm}^{2}$ ). This intensity of fluorescence decreased significantly at $1 \mathrm{~d}\left(10,570 \times 10^{6} \pm 4,173 \times 10^{6} /\right.$ photons $\left./ \mathrm{sec} / \mathrm{cm}^{2}\right)$, then decreased gradually from 1 to $28 \mathrm{~d}$. The fluorescence intensity of Cy5.5-labeled HA-polymers in the lung increased gradually after administration and showed the highest level at $2 \mathrm{~h}\left(4,885 \times 10^{6} \pm 1825 \times 10^{6} / \mathrm{photons} / \mathrm{sec} / \mathrm{cm}^{2}\right)$, then decreased gradually until $28 \mathrm{~d}$.

In the testis (Fig. 4E), the fluorescence intensity of Cy5.5labeled HA-NPs showed the highest level at $2 \mathrm{~h}\left(9,601 \times 10^{6}\right.$ $\left.\pm 4,327 \times 10^{6} / \mathrm{photons} / \mathrm{sec} / \mathrm{cm}^{2}\right)$, and this was generally maintained until $4 \mathrm{~h}$. This intensity of fluorescence decreased significantly at $1 \mathrm{~d}\left(4,499 \times 10^{6} \pm 2,936 \times 10^{6} / \mathrm{photons} / \mathrm{sec} / \mathrm{cm}^{2}\right)$ and decreased gradually from 1 to $28 \mathrm{~d}$. The fluorescence intensity of Cy5.5-labeled HA-polymers in the testis increased gradually after administration, showing the highest level at $4 \mathrm{~h}\left(3,802 \times 10^{6} \pm 1,765 \times 10^{6} / \mathrm{photons} / \mathrm{sec} / \mathrm{cm}^{2}\right)$, then gradually decreasing until $28 \mathrm{~d}$.

In the lymph node (Fig. 4F), the fluorescence intensity of HA-polymers was generally high within the designated period compared to that of HA-NPs. The fluorescence intensity of Cy5.5-labeled HA-NPs showed the highest level at $0.5 \mathrm{~h}$ $\left(5,188 \times 10^{6} \pm 265 \times 10^{6} / \mathrm{photons} / \mathrm{sec} / \mathrm{cm}^{2}\right)$, then decreased significantly at $2 \mathrm{~h}\left(2,430 \times 10^{6} \pm 57 \times 10^{6} / \mathrm{photons} / \mathrm{sec} / \mathrm{cm}^{2}\right)$, after which it increased again at $4 \mathrm{~h}\left(3,358 \times 10^{6} \pm 341 \times 10^{6} /\right.$ photons $/ \mathrm{sec} / \mathrm{cm}^{2}$ ). This intensity of fluorescence decreased significantly at $1 \mathrm{~d}\left(1,262 \times 10^{6} \pm 533 \times 10^{6} / \mathrm{photons} / \mathrm{sec} / \mathrm{cm}^{2}\right)$, then decreased gradually from 1 to $28 \mathrm{~d}$. The fluorescence intensity of Cy5.5-labeled HA-polymers in the lymph nodes was highest at $2 \mathrm{~h}\left(3,737 \times 10^{6} \pm 231 \times 10^{6} / \mathrm{photons} / \mathrm{sec} / \mathrm{cm}^{2}\right)$, and was generally maintained at a high level until $4 \mathrm{~h}$. This intensity of fluorescence decreased significantly at $1 \mathrm{~d}\left(1,272 \times 10^{6}\right.$ $\pm 55 \times 10^{6} /$ photons $\left./ \mathrm{sec} / \mathrm{cm}^{2}\right)$, then decreased gradually from 1 to $28 \mathrm{~d}$.

\section{Discussion}

The continuous development of new drug delivery agents is facilitated by the necessity to improve therapeutic effects while reducing negative side effects [19]. The potential effects of self-assembled polymeric NPs in tumor treatment have been investigated because they can surround a quantity of water-insoluble anticancer drugs and release them in a sustainable manner in their target tissues [3]. In recent years, HA has attracted tremendous interest in the field of drug delivery agents, and they have been used in various types of NPs, as well as ligands to prepare nanoplatforms for actively targeting agents, genes, and diagnostic agents [1]. HA modified macromolecular prodrugs and nano-scaled drug delivery agents showed improved target locating ability and therapeutic effects compared to free anticancer drugs [4]. However, as previously described, the time-dependent biodistribution and accumulation of HA are not yet completely understood.

In the present study, Cy5.5-labeled HAs were intravenously administrated into mice and the amount of fluorescence was measured in the main organs to investigate the biodistribution of HA as a drug delivery agent. Thereafter, the distributions of the HAs were monitored as a function of time using in vivo and ex vivo imaging systems.

To identify tissue distribution in real time, a dose of $5 \mathrm{mg} /$ $\mathrm{kg}$ of Cy5.5-labeled HA-NPs or HA-polymers was intravenously administrated into mice. In vivo imaging of the whole 
body showed that the level of fluorescence in HA-NPs (Fig. 1A) was much greater than that of HA-polymers (Fig. 1B) overall. These results suggest that particle size impacts the distribution and accumulation of NPs through the body. These findings are also supported by those of previous studies that showed NPs have much better intracellular absorption than microparticles, and that the effectiveness of absorption of $100 \mathrm{~nm}$ size particles was much better than that of larger size microparticles $(1-10 \mu \mathrm{m})[7,8,18]$. Although HA-NPs were labeled by 1.5 times more Cy5.5 than HApolymers, the level of fluorescence in the liver was at an even higher state in the HA-NPs group than the HA-polymers group. These results indicate that specific receptors for HA in the liver are molecular weight dependent and show much higher affinity to small-sized particles than large-sized particles [22]. In the current study, in vivo imaging showed that the liver and urinary bladder had much stronger fluorescence than other organs in both groups. These findings suggest that the liver and kidney are the main organs related to excretion of HA $[10,13]$.

Although the whole body images showed significant differences compared to the normal group (Fig. 1), distributions among organs were not clearly confirmed. The in vivo imaging to determine tissue distribution was not accurate and reliable because Cy5.5 was not strong enough to distinguish distribution among organs. Moreover, skin permeability, location of organs from the ventral part, or depth from the abdomen, and interference by fluorescence from blood and the surrounding organs made it difficult to distinguish organs. To identify more accurate sequential changes in HA-NPs and HA-polymers on the whole body image, more Cy5.5 labeled HA is needed; however, this may change the physicochemical properties of HA and the drugs it encapsulates. Therefore, this result (in vivo imaging) has to be supported by ex vivo imaging as discussed below.

Following administration of the Cy5.5-labeled HA-NPs or HA-polymers into the tail vein, the fluorescence intensities $\left(10^{6}\right.$ photons $\left./ \mathrm{sec} / \mathrm{cm}^{2}\right)$ were obtained from ex vivo images (Fig. 2). The change in fluorescence intensity was similar to that observed on ex vivo images, but areas non-detected on images were expressed by an imaging program with high discernment. The intensity of HA-NPs fluorescence was generally higher than that of HA-polymers (Figs. 3 and 4). Similarly, as described previously, small-sized particles can penetrate blood vessels and accumulate in tissues more easily than large-sized particles because of differences in receptor affinity $[7,8,18,22]$. However, in the lymph nodes (Fig. 4F), the fluorescence intensity of HA-polymers was generally higher than that of HA-NPs. Because of the atypical physiology of the lymphatic system, intravenously injected NPs do not lead to remarkable delivery to lymphatics [23]. Moreover, lymph node absorption of HA-NPs is highly dependent on density, not particle size; specifically, the absorption of HA-modified agent in the popliteal and iliac lymph nodes was found to mainly rely on HA-density [9]. In addition to particle size, other factors such as density influence distribution through lymphatics. Therefore, further studies are needed to identify the pharmacokinetics of NPs in the lymphatic system. For example, the effects of density and particle size should be investigated.

The fluorescence of HA-NPs and HA-polymers in the liver, spleen, lung, kidney, testis and lymph node showed much higher intensities than other organs (Fig. 4). Regardless of HA particle size, the fluorescence in each organ was maintained at a high level for $4 \mathrm{~h}$ and decreased at $1 \mathrm{~d}$, after which it was maintained at a low level that gradually decreased for up to $28 \mathrm{~d}$. Therefore, it took about $4 \mathrm{~h}$ for HA to distribute through the whole body and about one day to be metabolized and excreted from the animal body. However, some HA that accumulated in tissues appeared to need more time to be metabolized and excreted from body. In particular, regardless of HA particle size, the intensity of fluorescence in the liver was highest after intravenous injection, at which time it was also high in the spleen, kidney, lung, lymph node and testis (Fig. 3). One mechanism for the removal of HA from blood is by means of the HA receptor for endocytosis, which is primarily located in sinusoidal endothelial cells of the liver and spleen [22]. Given Teijeiro's report [22] and the finding that the liver had the highest fluorescence intensities, HA must be metabolized in liver. These results are also in accordance with those of our in vivo imaging. The spleen also showed higher fluorescence intensities than other organs, which was due to the reticuloendothelial system (RES) that takes up HA from the plasma and tissue fluid [9]. In short, the injected NPs were opsonized by plasma proteins in the blood stream, which was followed by their infiltration by RES in the liver and spleen [10, 16]. Similarly, the kidney also showed high fluorescence intensities. Therefore, these results imply that circulating nano-sized agents may be dissociated and degraded in vivo and excreted by renal clearance which was further supported by the results of in vivo imaging $[10,13]$. The lung was the major organ that showed high intensity because phagocytosis activities such as those conducted by alveolar macrophages contribute to redistribution of nanomaterials within the body [26]. Conversely, while the level of fluorescence decreased gradually in some organs such as liver (Fig. 4A) and spleen (Fig. 4B) at one day after intravenous injection, it suddenly increased again at $7 \mathrm{~d}$, then decreased again. This may have been because of phagocytic activity such as RES or due to excretion of HA that had accumulated in the tissues during $7 \mathrm{~d}$ [13].

Ex vivo imaging of total organs (Fig. 2) revealed that although HA must be metabolized and excreted by the liver and kidney, it showed continuous fluorescence in the liver, spleen and kidney until $28 \mathrm{~d}$, particularly for HA-NPs. Likewise, free Cy5.5 showed a similar pattern compared to Cy5.5-labeled HA-NPs and polymers. These findings indicate that free Cy5.5 was rapidly absorbed in the liver and maintained at a high concentration in the kidney, which gradually excreted it from the body. Cy5.5-labeled HAs and free 
Cy5.5 showed similar patterns, indicating that more experiments are needed to determine if HA is really metabolized in the liver and excreted from the kidney during $28 \mathrm{~d}$. Some studies have reported that nanomaterials do not always distribute in tissue according to particle size because they change their physicochemical properties during blood circulation [10]. Therefore, other test methods such as nuclear magnetic resonance are needed to identify physicochemical properties of nanomaterials in the liver and kidney. It is also necessary to compare these results with those obtained using other test methods that were not used in this experiment.

HA is a naturally existing material that largely constitutes the extracellular matrix of connective tissues and the vitreous body of the eye [25]. Furthermore, it is a biodegradable, biocompatible, nontoxic, and non-immunogenic component [2]. Many studies have shown that HA has no side effects such as cytotoxicity, neurotoxicity, mutagenicity, or reproductive toxicity. Moreover, histopathological analysis showed no pathological lesions in target organs, including the liver, spleen, kidney, lung, testis and epididymis (data not shown). Therefore, HA is likely to be a safe material for DDS.

In conclusion, in this study, comparative biodistributions and time-dependent fates of Cy 5.5-labeled HA-NPs and HA-polymers as a potential drug delivery agent were investigated in this study. The results showed that HA-NPs had much wider distribution than HA-polymers, implying that the small HA-NPs are able to reach the target site more effectively than large HA-polymers. In the liver, spleen and kidney, much of the HA was distributed during the first $4 \mathrm{~h}$, after which it was suddenly excreted at $1 \mathrm{~d}$ and then gradually excreted until $28 \mathrm{~d}$. These findings imply that the liver and spleen were the main organs that metabolize HA and that $\mathrm{HA}$ is excreted from the body through kidney. In the final analysis, HA that circulates in the blood stream is absorbed by the mononuclear phagocytic system in the spleen and liver, metabolized by the liver and excreted from the body through renal clearance. However, some HA that are absorbed by alveolar macrophages are redistributed throughout the lung and HA accumulated by RES or tissue is recirculated at around $7 \mathrm{~d}$. This information on distributions of HA-NPs and HA-polymers in the body may have important clinical implications regarding the safety of self-assembled HA nanoparticles, which can be widely used in biomedical applications.

\section{Acknowledgments}

This research was supported by the Basic Science Research Program (2016R1D1A1B01015625) through the National Research Foundation of Korea (NRF) funded by the Ministry of Education, Science.

\section{References}

1. Arpicco S, Milla P, Stella B, Dosio F. Hyaluronic acid conjugates as vectors for the active targeting of drugs, genes and nanocomposites in cancer treatment. Molecules 2014, 19, 3193-3230.

2. Battistini FD, Olivera ME, Manzo RH. Equilibrium and release properties of hyaluronic acid-drug complexes. Eur $\mathrm{J}$ Pharm Sci 2013, 49, 588-594.

3. Choi KY, Chung H, Min KH, Yoon HY, Kim K, Park JH, Kwon IC, Jeong SY. Self-assembled hyaluronic acid nanoparticles for active tumor targeting. Biomaterials 2010, 31, 106-114.

4. Choi KY, Min KH, Na JH, Choi K, Kim K, Park JH, Kwon IC, Jeong SY. Self-Assembled hyaluronic acid nanoparticles as a potential drug carrier for cancer therapy: synthesis, characterization, and in vivo biodistribution. J Mater Chem 2009, 19, 4029-4280.

5. Choi KY, Min KH, Yoon HY, Kim K, Park JH, Kwon IC, Choi K, Jeong SY. PEGylation of hyaluronic acid nanoparticles improves tumor target ability in vivo. Biomaterials 2011, 32, 1880-1889.

6. Collins MN, Birkinshaw C. Hyaluronic acid based scaffolds for tissue engineering-a review. Carbohyd Polym 2013, 92, 1262-1279.

7. Desai MP, Labhasetwar V, Amidon GL, Levy RJ. Gastrointestinal uptake of biodegradable microparticles: effect of particle size. Pharm Res 1996, 13, 1838-1845.

8. Desai MP, Labhasetwar V, Walter E, Levy RJ, Amidon GL. The mechanism of uptake of biodegradable microparticles in Caco-2 cells is size dependent. Pharm Res 1997, 14, 1568-1573.

9. Fraser JRE, Appelgren LE, Laurent TC. Tissue uptake of circulating hyaluronic acid: a whole body autoradiographic study. Cell Tissue Res 1983, 233, 285-293.

10. Hwang HY, Kim IS, Kwon IC, Kim YH. Tumor targetability and antitumor effect of docetaxel-loaded hydrophobically modified glycol chitosan nanoparticles. J Control Release 2008, 128, 23-31.

11. Joris F, Manshian BB, Peynshaert K, De Smedt SC, Braeckmans K, Soenen SJ. Assessing nanoparticle toxicity in cell-based assays: influence of cell culture parameters and optimized models for bridging the in vitro-in vivo gap. Chem Soc Rev 2013, 42, 8339-8359.

12. Lee H, Lee K, Kim IK, Park TG. Synthesis, characterization, and in vivo diagnostic applications of hyaluronic acid immobilized gold nanoprobes. Biomaterials 2008, 29, 47094718.

13. Lee JS, Ji HJ, Chai H, Jeong JH, Nam SY, Yun YW, Lee BJ. Distribution and accumulation of Cy5.5-labeled hydrophobically modified glycol chitosan in mice. J Prev Vet Med 2015, 39, 48-57.

14. Marquis BJ, Love SA, Braun KL, Haynes CL. Analytical methods to assess nanoparticle toxicity. Analyst 2009, 134, 425-439.

15. Mironov V, Kasyano V, Shu XZ, Eisenberg C, Eisenberg L, Gonda S, Trusk T, Markwald RR, Prestwich GD. Fabrication of tubular tissue constructs by centrifugal casting of cells suspended in an in situ crosslinkable hyaluronangelatin hydrogel. Biomaterials 2005, 26, 7628-7635.

16. Moghimi SM, Hunter AC, Murray JC. Long-circulating and target-specific nanoparticles: theory to practice. Pharmacol Rev 2001, 53, 283-318.

17. Nam JM, Thaxton CS, Mirkin CA. Nanoparticle-based bio- 
bar codes for the ultrasensitive detection of proteins. Science 2003, 301, 1884-1886.

18. Panyam J, Labhasetwar V. Biodegradable nanoparticles for drug and gene delivery to cells and tissue. Adv Drug Deliv Rev 2003, 55, 329-347.

19. Rösler A, Vandermeulen GWM, Klok HA. Advanced drug delivery devices via self-assembly of amphiphilic block copolymers. Adv Drug Deliv Rev 2001, 53, 95-108.

20. Song U, Jun H, Waldman B, Roh J, Kim Y, Yi J, Lee EJ. Functional analyses of nanoparticle toxicity: a comparative study of the effects of $\mathrm{TiO}_{2}$ and $\mathrm{Ag}$ on tomatoes (Lycopersicon esculentum). Ecotoxicol Environ Saf 2013, 93, 60-67.

21. Taton TA, Mirkin CA, Letsinger RL. Scanometric DNA array detection with nanoparticle probes. Science 2000, 289, $1757-1760$

22. Teijeiro C, McGlone A, Csaba N, Garcia-Fuentes M, Alonso MJ. Polysaccharide-based nanocarriers for drug delivery. In: Torchilin V (ed.). Handbook of Nanobiomedical Research: Fundamentals, Applications and Recent Developments. Vol. 1. 1st ed. pp. 235-278, World Scientific, Singapore, 2014.

23. Thomas SN, Schudel A. Overcoming transport barriers for interstitial-, lymphatic-, and lymph node-targeted drug delivery. Curr Opin Chem Eng 2015, 7, 65-74.

24. Tiantian Y, Wenji Z, Mingshuang S, Rui Y, Shuangshuang S, Yuling M, Jianhua Y, Xinggang Y, Shujun W, Weisan P. Study on intralymphatic-targeted hyaluronic acid-modified nanoliposome: influence of formulation factors on the lymphatic targeting. Int J Pharm 2014, 471, 245-257.

25. Tripodo G, Trapani A, Torre ML, Giammona G, Trapani G, Mandracchia D. Hyaluronic acid and its derivatives in drug delivery and imaging: recent advances and challenges. Eur J Pharm Biopharm 2015, 97, 400-416.

26. Walkey CD, Olsen JB, Guo H, Emili A, Chan WCW. Nanoparticle size and surface chemistry determine serum protein adsorption and macrophage uptake. J Am Chem Soc 2012, 134, 2139-2147.

27. Zhang H, Ji Z, Xia T, Meng H, Low-Kam C, Liu R, Pokhrel S, Lin S, Wang X, Liao YP, Wang M, Li L, Rallo R, Damoiseaux R, Telesca D, Mdler L, Cohen Y, Zink JI, Nel AE. Use of metal oxide nanoparticle band gap to develop a predictive paradigm for oxidative stress and acute pulmonary inflammation. ACS Nano 2012, 6, 43494368 